\title{
Capturing of the internal mechanics of liquid-granular flows comprised of polydisperse spherical particles
}

\author{
Devis Gollin ${ }^{1, \star}$ and Elisabeth Bowman ${ }^{1, \star \star}$ \\ ${ }^{1}$ The University of Sheffield, Department of Civil \& Structural Engineering, Western Bank, Sheffield, S10 2TN, UK
}

\begin{abstract}
This paper presents a series of flume experiments designed to examine the motion and arrest of concentrated granular-fluid flows, with a view to understanding the role of polydispersity in debris flows. A non-intrusive technique is used to investigate the internal behaviour of small scale experimental flows. Three different particle size distributions comprised of polydisperse spherical particles and one with the finer component made of angular particles were analysed. The choice of using spherical shaped particles was made to improve the visualization of the internal mechanics without reducing overmuch the complexity involved in the study of these flows. We examined and compared the internal velocities of the flows and their depositional spreads. While the optical performance of the non-intrusive technique was improved, some of the characteristics commonly seen in these types of granular flows were not observed. Velocity profiles obtained in the body of the flows were similar in shape but with differences in velocity magnitude depending on the amount of fines and the angularity of the particle in one case. Depositional runouts between flows were similar at low inclinations when little internal energy was supplemented to the system or when the viscous effects dominated the mechanics at steeper angles.
\end{abstract}

\section{Introduction}

Debris flows constitute a significant natural hazard in regions of mountainous terrain. They are often described as a succession of non-uniform surges with particle sizes ranging from clay to boulders. Flow segregation can occur where larger particles are preferentially transported to the snout which is characterized by a reduced water content and greater friction resistance to motion than the dispersion behind it. The rear interior, where large amount of suspended fines tend to accumulate, provides a mobile core which advects some of its downstream momentum into the resistive, coarse-grained snout, thereby providing the force to drive the snout forward. This mixture of sediment stops when approaching gently sloping surfaces. These areas of the deposit spread or runout are generally of most interest in terms of human risk. How far such flows will travel is critical for delineating areas at risk and to design protective measures.

The inherent complexity and the multitude of heterogeneities within granular flows make them very hard to study in the field. However, fundamental characteristics of mobile granular flows can be reproduced in laboratory experiments, albeit with some simplifications. This has shed some light on their overall behaviour, but the mechanics of their movement is still far from understood. Hence debris flows and more in general granular flows are subject of in-

${ }^{\star}$ e-mail: devis.gollin@sheffield.ac.uk

$\star \star$ e-mail: e.bowman@sheffield.ac.uk tense research from an experimental perspective which can also be supported by numerical and theoretical analyses

The combined effects of mixture composition and problem geometry on internal behaviour and the effects on the runout can be reproduced in laboratory flumes. Such an approach was previously adopted by Sanvitale and Bowman [1] . Several flows were studied using an innovative non-intrusive technique that allowed the visualization of a transparent medium, thus the internal dynamics of a flow, while matching closely the properties of real debris flows. Here a similar apparatus was used, but with modifications for this particular work. Based on the previous results, more simplified solid particles are employed in order to improve the visualisation technique while trying to keep an approximation to debris flow behaviour. The choice of the particles leads to a better visualization of the flow interior. However, we show that there are fundamental limitations on the choice of the shape and size of particles.

\section{Background}

Iverson [2] has argued that the dynamic similarity between natural and small-scale debris flows is probably unattainable since small models cannot reproduce the same stresses as those of real large debris flows. However, Bowman and Sanvitale [3] showed that comparison between the two scales does appear feasible and careful laboratory testing performed on small scale flumes can still inform on the mechanics behind debris flow behaviour. It was shown that the well-graded nature of debris flow is not 


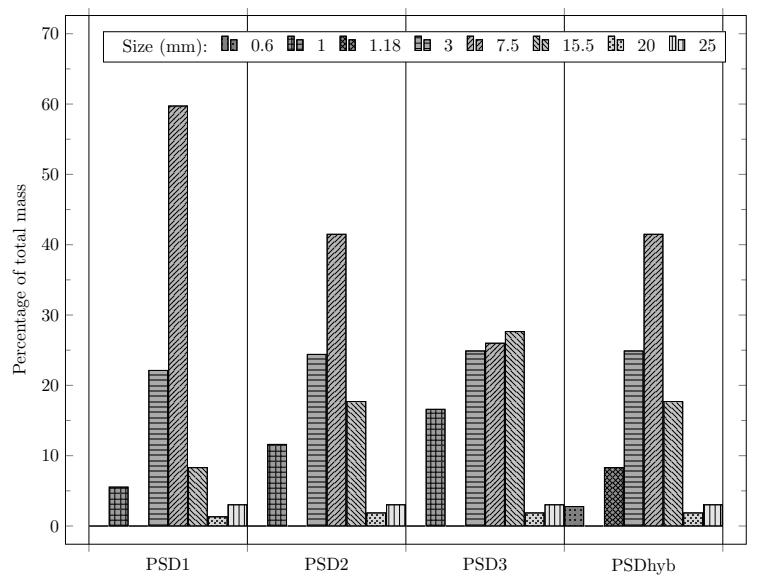

Figure 1: Particle size distributions

incidental, but an important factor affecting their mobility and the capacity to travel far before the final deposition phase. The correct choice of the particle size distribution (PSD) enabled important aspects of debris flow behaviour to be replicated even at small scale, such as the effects of flow segregation and its influence on final deposit shape and grading profile.

An experimental debris flow consisting of well-graded materials may generate low frictional resistance within the flow interior as the flow segregates with agitated finer particles reducing the propensity for fluid to exit from the mass. Hence pore pressures may be greater than hydrostatic and effective stress greatly reduced. This is different from typical experiments characterized by a relatively uniformly-graded particle size distribution. In this case segregation cannot occur and the mixtures maintain a steady frictional resistance. The fluid is evenly distributed throughout the flow so that momentum is lost quickly due to the uniformly distributed friction resistance and the reduced effect of pore pressure. Comparatively, for systems with the same masses of solids and fluid, well-graded mixtures show higher velocity, smaller flow thickness and a larger runout extent than uniform flows [4].

These distinct behaviours may explain the difference in behaviour at large and small scale that is often observed. If well-graded materials are used for small scale work, high overall mobility can be achieved in this configuration despite the lower velocity attained.

Here small scale experiments considering the flow of transparent materials down an inclined chute are described. These are characterized by particle size distributions made of few, but distinctly different, particle diameters in the attempt to step away from monodisperse realizations but at the same time, only gradually increase the complexity of the investigations.

\section{Experimental set-up}

The flume used in this study is similar to that used by Sanvitale and Bowman [1]. The apparatus is a sloping rectangular $15 \mathrm{~cm}$ wide and $200 \mathrm{~cm}$ long channel that can tilt from horizontal up to $45^{\circ}$. At the upstream end, the material is held in a rectangular tank. A double-slider gate mechanism enables control of the releasing flow height that develops downstream. Attached at the bottom of the inner gate is a flexible flap with holes that holds a small pocket of liquid. At the opening of the gate this liquid exits first removing any entrainment of air bubbles which would be otherwise trapped in the solid phase and carried down the channel, thus creating unwanted visible micro lenses in the images and interfering with the laser plane. The rough base has been made via $3 \mathrm{D}$-printing using a plastic material (Nylon SLS). Semi-spherical particles of diameter $3 \mathrm{~mm}$ at the circular edge are organized in a hexagonal pattern and positioned at the bottom. Near the end of the flume a planar laser light is shone $50 \mathrm{~mm}$ away from the sidewall in order to illuminate the transparent material. At the same fixed location measurements were taken for all flows. The lit surface of the granular flow is captured by a high-speed camera (Phantom Miro 310) at a frame rate of 500 fps. A typical example of images for these particular flows is shown in Fig 2. Once collected, the images are processed via an open-source particle image velocimetry (PIV) software [5] and velocity profiles extracted for each flow. A flat deposition area is situated at the end of the channel where the material is collected and measurements of final runout length and area recorded.

The visualization of the flow interior is done via planer laser induced fluorescence (PLIF), which requires the matching of the solid and liquid refractive indices (RI). A fully saturated flow is required for this method to work properly. The materials used are crushed angular particles borosilicate glass with size 0.6 and $1.18 \mathrm{~mm}$, respectively, and spherical particles of the same material with diameters ranging from 1 to $25 \mathrm{~mm}$. Given the focus was on image quality here, manufactured spherical particle which are nearly flawless, enabled an improvement to that achieved previously using angular glass particles. However, the cost of very fine spherical particles was prohibitive, hence fines were made of angular materials. The fluid (a hydrocarbon oil produced by Cargille laboratories) was refractively matched to the glass and a small amount of fluorescent dye (Nile Red) added so that to distinguish the particles from the brighter background. All tests were conducted at a controlled room temperature of $23^{\circ}$. This was found optimal for matching the two refractive indices as the RI for the liquid varies with the temperature.

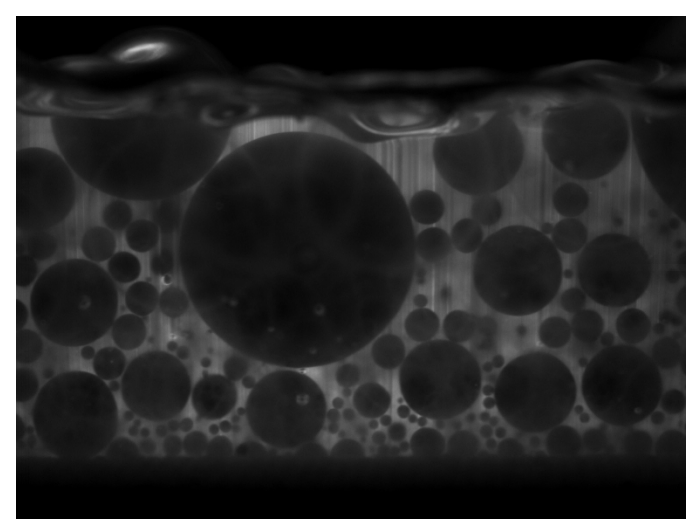

Figure 2: Internal flow image for PSD1 at $15^{\circ}$ 


\subsection{Methodology}

All tests were carried out with the same experimental conditions. The opening height of the gate was kept constant. Three chute inclinations were used, respectively, $15^{\circ}, 20^{\circ}$ and $25^{\circ}$. A total mass of $18 \mathrm{Kg}$ for each mixture was loaded inside the hopper and then saturated so that the initial sediment volumetric concentration (Cs) was 0.57 (i.e. porosity $=0.43$ ). The four particle size distributions (PSD) used in this study are shown in Fig 1. They are presented as histograms showing the percentage of mass for each particle size over the total mass. The smallest and largest sizes were limited due to practical reasons, however we are able to approximate those used by Sanvitale and Bowman [1] (in Fig. 8). PSD1, PSD2 and PSD3 are composed of spherical particles only. For each of these three we progressively increased the amount of finer materials $(1 \mathrm{~mm})$ from $5.53 \%$ for PSD1, $11.2 \%$ for PSD2 and $16.6 \%$ for PSD3, reduced the number of particle in the middle of the distribution $(7.5 \mathrm{~mm})$ and increased the number of larger particles $(15.5 \mathrm{~mm})$. The two largest sizes, 20 and $25 \mathrm{~mm}$ were kept constant throughout due to the limited number of particles available. PSD $_{h y b}$ is the same as PSD2 but all $1 \mathrm{~mm}$ particles (i.e. $11.2 \%$ of the total mass) were substituted by angular particles of 0.6 and $1.18 \mathrm{~mm}$ in order enhance expected pore pressure effects caused by addition of fines.

\section{Results}

For the flows released at an inclination of $15^{\circ}$, velocity profiles obtained by averaging over the body of the flows

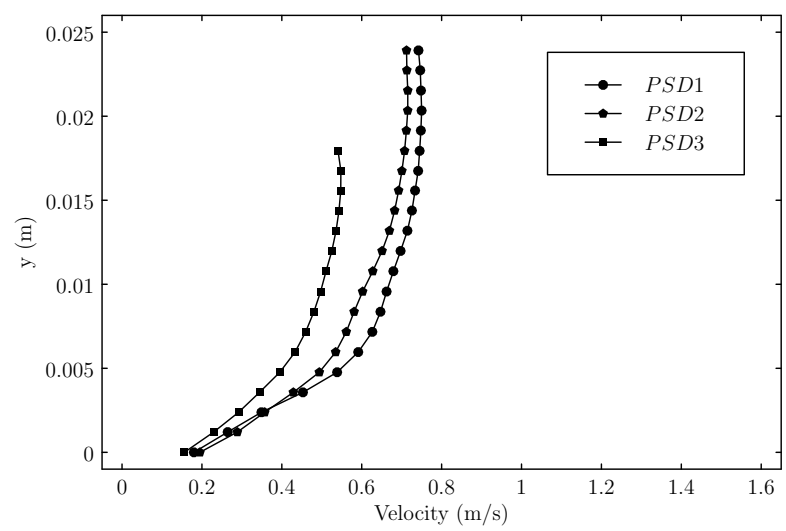

Figure 3: Velocity profiles for flows released at $15^{\circ}$

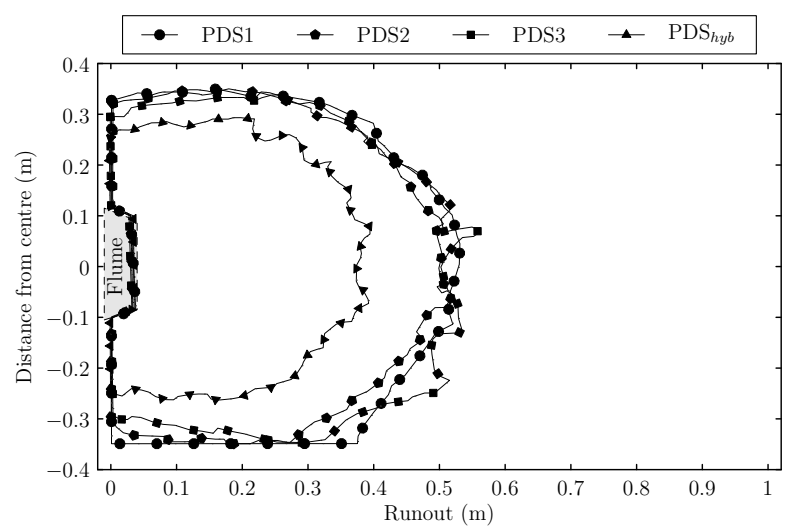

Figure 4: Depositional runouts for flows released at $15^{\circ}$ (i.e. avoiding the noisy front and the tail of the surge) are shown in Fig. 3. In all cases a slip velocity developed at the bottom boundary. PSD1 and PSD2 have similar flow heights and velocity magnitudes compared to PSD3. PDS $S_{h y b}$ was not able to develop sufficient momentum which resulted in a thick layer of material being deposited over the channel. Only a short recording was possible before the material stopped, which did not allow a velocity profile to be determined.

From the analysis of flow images it was revealed that after the coarser but still saturated front smaller particles segregated to the bottom of the flow leaving larger ones to travel at higher velocities (e.g. Fig. 2). This occurred for all inclinations. In addition, fine materials (smaller than 1 and $1.18 \mathrm{~mm}$ ) were partially trapped and slowed by the rough base made of $3 \mathrm{~mm}$ semi-spheres.

The profiles of the final deposit are shown in Fig 4. These were similar to each other for PSD1, PSD2 and PSD3. If the velocities are compared to the runout lengths, some changes would be expected for the slower PDS3. However, the length and shape of the spreads were very similar indicating that not enough inertial energy was induced into the system and that the material was progressively accumulating without showing characteristic depositional behaviour. This was different for $\mathrm{PSD}_{h y b}$, possibly due to the increased frictional resistance provided by the angular fine particles leading to an even shorter runout. Note that the choice to use $15^{\circ}$ was led by previously tested flows (not shown here) conducted with monodisperse 3 $\mathrm{mm}$ dispersions which developed much higher velocities.

To increase the energy in the system and produce different behaviours, the inclination needed to be increased. While $20^{\circ}$ showed intermediate behaviours between $15^{\circ}$ and $25^{\circ}$, here we focus on flows released at $25^{\circ}$ only. Velocity profiles obtained from the body of the flows are depicted in Fig. 5. Similarly to the flows at $15^{\circ}$, a distinct slip velocity was generated at the bottom boundary. For PSD1, PSD2 and PSD3 the magnitudes of this quantity decreased progressively with an increasing amount of fines. PDS $_{h y b}$ travels slower than PSD3 due to the increased friction. Note that the all profiles tend to reverse back at the free surface (i.e. with slower velocities than the middle part), which is unexpected for these flows, in which we would expect a maximum at the free surface. This was caused by the changing of the saturation line (also the limit for the PLIF technique). For these faster flows, occasional and local reduction of the saturation line is caused by the crossing of big particles transported at the free surface. These are interpreted by PIV as areas with lower velocity. Hence averaging results in an apparent reversing of the velocity profile at the top of the flow. This was also noticed when the average was done for shorter periods of time (e.g. for 30 or 50 frames only).

The flows analysed at an inclination of $25^{\circ}$ produced the depositional areas shown in Fig. 6. PSD1 had the longest runout and fines segregated close to the bottom boundary. This resulted in larger particles dominating the mechanics during runout producing a longer spread. Conversely, from the analysis of flow images for PSD2 and 


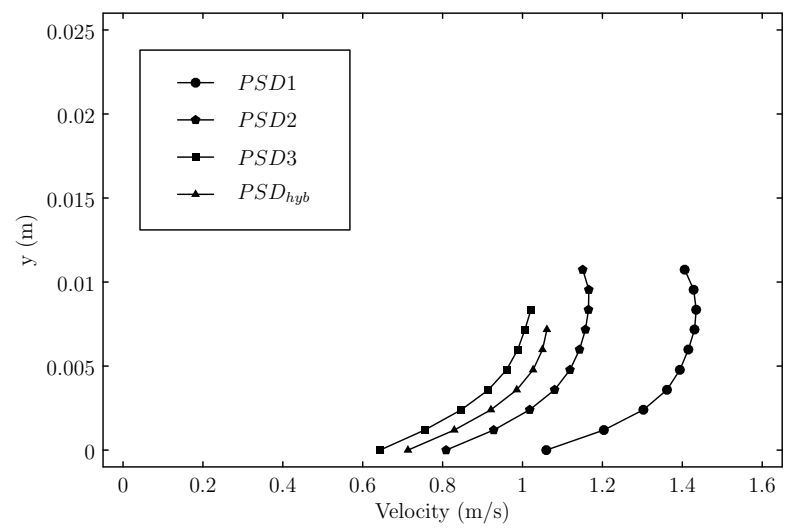

Figure 5: Velocity profiles for flows released at $25^{\circ}$

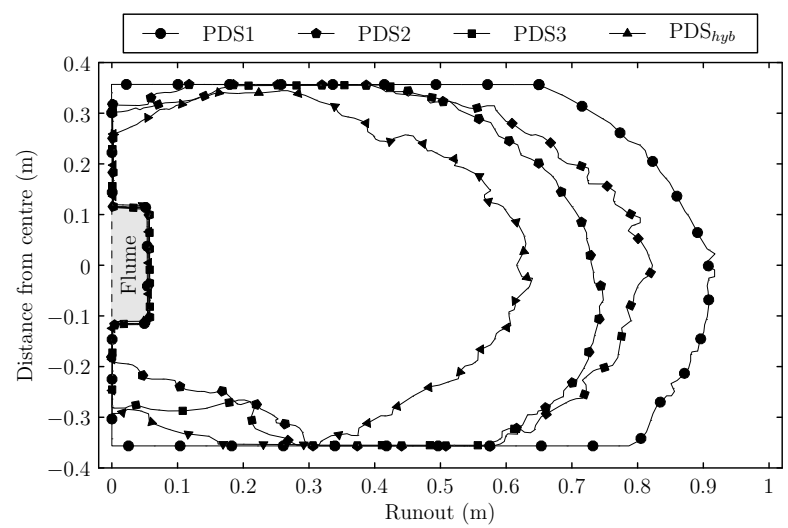

Figure 6: Depositional runouts for flows released at $25^{\circ}$

PSD3 it was clear that fine particles were more evenly distributed throughout the flow height. They have shorter and comparable runouts, with PSD3 being somewhat longer. That is, increasing the amount of fine particles $(<1 \mathrm{~mm}$ for these three PSDs) considerably slowed the flows. Therefore, the expected changes of runout caused by adding fines to increase pore pressure (i.e. via accumulation of fines in the body and rear) did not occur, instead viscous and vertical segregation effects appeared to dominate. Again, $\mathrm{PDS}_{\text {hyb }}$ produced shortest runout due to the increased frictional resistance of fine angular particles.

\section{Conclusion}

This paper presented the results obtained for polydisperse granular flows characterized by different particle size distributions and particle shapes. Velocity profiles were obtained from internal cross sections of the flows. The use of spherical particles allowed us to improve the optical performance of the PLIF technique. However, some of the typical depositional characteristics for these types of granular flows were not observed. The extents of the runout areas were similar for low energy flows at an inclination of $15^{\circ}$. In contrast, for $25^{\circ}$ it was clear that flows with the highest amount of fines were affected by strong viscous effects and vertical segregation. For both inclinations, $\mathrm{PSD}_{h y b}$ (a modified PSD2 with angular particles) had the shortest runout due to increased fictional resistance.

Perhaps the display of different depositional behaviours could be achieved by a more careful choice of the
PSD compositions. Moving from uniform to well-graded distribution could be done by varying the amount of fine and large particles around a specific point (e.g. a mean diameter $\left.\left(\mathrm{D}_{50}\right)\right)$, as done previously [1]. For these tests we were limited in the amount of large particles available while considerably increasing the amount of fines. That is, by just increasing the fines content we only induced additional viscous effects. In addition, large step changes in the specific particle sizes - e.g. beyond Terzaghi's filter limit [6] - may have enabled particle size segregation to spontaneously occur at the commencement of the flows. Finally, while we considered velocity profiles obtained in the body of the flow, it would be interesting to compare runout versus front velocity as in [3] but this velocity was difficult to obtain due to the controlled flow release adopted to improve the flow visualization.

Ultimately, other substantial effects remain to be investigated. In case of particle vertical segregation, frictional and viscous gradients develop across the flow. Local effects may be generated, for example, the variations of global bulk motion of the interstitial fluid relative to the granular material as well as different distribution of the stresses connected to the particle rearrangement. The degree of segregation and the related effects may be quantified by estimating solid concentration profiles. The bottom also plays an important role. Very bumpy base may increase the frictional resistance due to small particles trapped in the gaps at shallow angles but also generate strong agitation and remixing at stepper ones. To clarify the boundary effect, the same flows should be tested at different bottom boundary. Undertaking further investigations could lead to new insights into internal and depositional mechanics of these granular flows.

\section{Acknowledgements}

The first author is supported by an Engineering and Physical Sciences Research Council (EPSRC) DTA Scholarship.

\section{References}

[1] Sanvitale, $\mathrm{N}$ and Bowman, E.T., International Journal of Physical Modelling in Geotechnics 12(4), 129-142 (2012)

[2] Iverson, R. M., Reviews of Geophysics 35(3), 245296 (1997)

[3] Bowman, E.T. and Sanvitale, N. 17th International Conference on Soil Mechanics and Geotechnical Engineering, 470-473 (2009)

[4] Sanvitale N., Bowman E.T., Genevois R. 5th International Conference on Debris-Flow Hazards Mitigation: Mechanics, Prediction and Assessment, 375-384 (2009)

[5] Thielicke, W. and Stamhuis, E.J. Journal of Open Research Software. 2(1), e30 (2014).

[6] Skempton, A. W. and Brogan J.M, Géotechnique. 44(3), 449-460(1994). 